\title{
Research on Improving Prediction Accuracy of Sports Performance by Using Glowworm Algorithm to Optimize Neural Network
}

\author{
Fan Zhang
}

\begin{abstract}
In order to improve the accuracy of sports performance prediction and solve the shortcomings of low precision and slow speed of current sports performance prediction model, this paper proposes a prediction model based on glowworm optimization neural network. Firstly, the training samples and test samples of the neural network are generated by pretreatment of the sports performance; secondly, the connection weights and thresholds of the BP neural network are determined by using the glowworm optimization algorithm, and the prediction model of the sports performance is established by learning the training samples; finally, the prediction effect is tested by specific simulation experiments. The results show that the glowworm optimized neural network improves the prediction accuracy of sports performance, and solves the limitations of other sports performance prediction models. The prediction results are more reliable, which can provide scientific decision-making basis and valuable information for sports training.
\end{abstract}

Index Terms-Sports performance, glowworm algorithm, neural network, physical fitness.

\section{INTRODUCTION}

With the continuous improvement of people's level, more and more sub-health phenomena appear in the body, especially the physical quality of college students is not as good as before. How to improve the physical quality of college students has aroused widespread concern in Chinese Mainland [1]. Sports performance prediction can describe the physical health of college students and athletes' competitive level, so sports performance modeling and prediction can make a relatively reasonable training plan for athletes and college students to improve sports performance [2], [3].

Sports performance data is regarded as a set of data sets, and multiple linear regression is used to model it. According to the parameters of an athlete's performance is estimated, but because of the multiple linear regression, all kinds of samples are distributed evenly, and presents a nonlinear growth trend.

Manuscript received September 2, 2018; revised December 9, 2018. This work was supported in part by the Project of the Fundamental Research Funds for the Central Universities under Grant LGZD201805, in part by the 13th Five-Year plan project of Jiangsu Education Science under Grant C-c/2018/01/11, in part by the Project of China Postdoctoral Science Foundation under Grant 2017M611849, in part by Jiangsu Qing Lan Project under Grant 2017, and in part by Pre-research project of Nanjing Forest police College under Grant LGY201603 , and in part by Nanjing Forest police College Teaching Reform Project under Grant ZD18104.

Fan Zhang is with College of Nanjing Forest Police, Nanjing, Jiangsu 210023 China. He is also with the Sports Science postdoctoral research station, Nanjing Normal University, Nanjing, Jiangsu 210023 China (e-mail: zhangfan@nfpc.edu.cn).
This situation is not consistent with the actual situation of sports performance data, and the prediction results are unreliable, the practical application value is not high [4]. In recent years, with the continuous development of fuzzy theory and grey theory, there are many nonlinear sports performance prediction models, which make the reliability of sports performance prediction better than multiple linear regression model [5]. In practical application, these models also have obvious limitations, such as fuzzy theory is difficult to grasp, requires a certain theoretical basis, and grey theory regards the sports performance prediction process as a black box, the predicted results can not be interpreted well.

Nowadays, some scholars have put forward the prediction model of sports performance based on neural network. Neural network has the ability of self-organization and non-linear mapping. It can describe the changing characteristics of sports performance better and establish a better prediction model of sports performance than other models [6], [7]. However, the relevant parameters of neural network such as threshold and weight have a great impact on the performance of neural network. If these parameters are not reasonably determined, the results of sports performance prediction will be very low. In order to solve the problem of determining the parameters of the neural network, some scholars have proposed to use genetic algorithm, particle swarm optimization and other methods to determine the threshold and weight of the neural network, which can effectively improve the prediction accuracy of sports performance. Genetic algorithm and particle swarm optimization belong to stochastic optimization algorithm. Generally, only sub-optimal solution can be found, and the global optimal solution can not be found. Moreover, it is easy to find the threshold and weight of local optimum, which makes the neural network learning time long, the network structure complex, and affects the sports performance modeling effect [8], [9]. Therefore, in order to improve the prediction accuracy of sports performance, this paper proposes a prediction model of sports performance based on glowworm algorithm [10] optimization neural network, in order to solve the above problems.

\section{NEURAL NETWORK AND GLOWWORM OPTIMIZATION ALGORITHM}

\section{A. Neural Network}

Among all the neural networks, BP neural network has faster learning speed, more flexibility and more versatility. It can infinitely approximate a nonlinear system and accurately 
describe the nonlinear characteristics of the system. Set the input of the system to $x(i), i=1,2, \cdots, n$. The calculation formula of hidden layer input vector and output vector of BP neural network is as follows:

$$
\begin{gathered}
S_{j}=\sum_{i=1}^{n} \omega_{i j} x(i)-\theta_{j} \\
b_{j}=\exp \left(\sum_{i=1}^{n} \omega_{i j} x_{i}-\theta_{j}\right)
\end{gathered}
$$

In the formula: ${ }^{\omega_{i j}}$ represents the connection weight between the input layer and the hidden layer; ${ }^{\theta_{j}}$ represents the threshold between the two.

The input vectors and output vectors of the output layer of the BP neural network are:

$$
\begin{gathered}
L=\sum_{j=1}^{p} \omega_{i k} b_{j}-\theta_{k} \\
x_{i+1}=\exp \left(\sum_{i=1}^{p} v_{j} b_{j}-\gamma\right)
\end{gathered}
$$

In the formula, ${ }_{j}$ denotes the connection weight between the hidden layer and the output layer of the neural network, and $\gamma$ denotes the threshold value between the hidden layer and the output layer of the neural network.

\section{B. Glowworm Optimization Algorithm}

Glowworm algorithm is an algorithm based on glowworm luminescence to attract other glowworms. glowworm position represents a solution of the problem. The luminous brightness is related to fitness function. The bigger the fitness value, the stronger the luminous brightness. Weak glowworms move toward brighter ones. With the increase of iteration times, most glowworms gather around the brightest position, which represents the optimal solution of the problem. Make the following assumptions:

1) all glowworms do not have sex. glowworm can attract other glowworms.

2) attraction is closely related to the brightness of glowworms. Weak glowworms usually move toward brighter glowworms, and the brightness is inversely proportional to distance.

3) brightness is directly determined by the fitness function of the problem.

$$
I=I_{0} e^{-\gamma r}
$$

In the formula: gamma is the absorption factor of light intensity; $\mathrm{R}$ is the distance between glowworms.

The formula of attraction is as follows:

$$
\beta=\beta_{0} e^{-\gamma r^{m}}
$$

In the formula: $\beta_{0}$ is the attraction of $r=0$.

Two glowworms are $i, j$, and satisfy the conditions: $I_{0}(i)>I_{0}(j)$, then $i$ is attracted by $j$, to update their position, namely:

$$
x_{i}(t+1)=x_{i}(t)+\beta_{0} e^{-\gamma r_{i j}^{2}}\left(x_{j}(t)-x_{i}(t)\right)+\partial \ni_{i}
$$

In the formula: $x_{i}(t), x_{i}(t+1)$ is the current position and the next moment position of $i$; ${ }^{i j}$ denotes the distance between $i$ and $j$; $\partial$ and ${ }^{{ }}{ }_{i}$ are random numbers.

From equation (7), it can be seen that the attractiveness directly affects the step size of the glowworm movement, while the $\beta_{0}$ and $\gamma$ of the basic glowworm algorithm remain unchanged and can not be adaptively changed, which makes the initial search ability poor, and later can not converge to the optimal solution. In order to prevent this problem, the attraction coefficient is improved and adaptively changed, as follows:

$$
\begin{gathered}
\beta_{0}=\left\{\begin{array}{l}
\beta_{\text {begin }} \times \sigma_{\beta}^{\text {itor }}, \quad \beta_{0}>\eta \\
\eta \quad \beta_{0} \leqslant \eta
\end{array}\right. \\
\gamma=\gamma_{\text {begin }} \times \sigma_{\gamma}^{\text {itor }}
\end{gathered}
$$

In the formula: $\sigma_{\beta}, \sigma_{r}$ indicates the range of the attenuation coefficient; itor represents the number of iterations. The change range of $\beta_{0}$ is $\left[\eta, \beta_{\text {begin }}\right]$, the initial value of $\beta_{0}$ is relatively large, the search range is large, the probability of finding the optimal solution is large, with the increase of the number of iterations, the attractiveness decreases, and the optimal position can be quickly found.

Intensity absorption factor $\gamma$ is regarded as the range of sight of glowworms. The larger the value, the smaller the range of sight, and vice versa. In the early stage, glowworms are only associated with the surrounding glowworms, which can effectively avoid falling into local optimum. In the later stage, the value of $\gamma$ becomes smaller, and the glowworms move to the optimum position.

\section{Prediction Model of Sports Performance Based ON NEURAL NETWORK OPTIMIZED BY GLOWWORM \\ ALGORITHM}

1) collect sports performance data and use formula (10) for processing:

$$
x_{i}=\left(x_{i}-x_{\min }\right) /\left(x_{\max }-x_{\min }\right)
$$

In the formula: ${ }^{x_{i}}$ is the result after treatment; ${ }^{x_{i}}$ is the 
original result; $x_{\max }, x_{\min }$ represents the maximum and minimum.

After the prediction, the results of the sports performance prediction are dealt with in the following way:

$$
x_{i}=x_{i} \times\left(x_{\max }-x_{\min }\right)+x_{\min }
$$

2) set the relevant parameters of the glowworm algorithm, such as the number of glowworms, the maximum number of iterations, etc.

3) randomly generate glowworm population, and each location vector corresponds to the threshold and weight of BP neural network. Fitness function value is obtained according to threshold value, weight value and training sample of sports achievement, and the prediction precision of sports achievement is selected as fitness function.

4) According to the fitness function, the location of glowworms was evaluated, and the weak attracted glowworms moved to the strong attracted position to update the location of all glowworms.

5) According to the fitness function value, we estimate the brightness of glowworm and search it locally.

6) If the end condition is satisfied, the globally optimal position of the glowworm is found, otherwise step 4) is returned to continue.

7) According to the global optimal position vector as the threshold and weight of BP neural network, the sports performance prediction model is established.

In summary, the workflow of the sports performance prediction model based on glowworm optimization neural network is shown in Fig. 1.

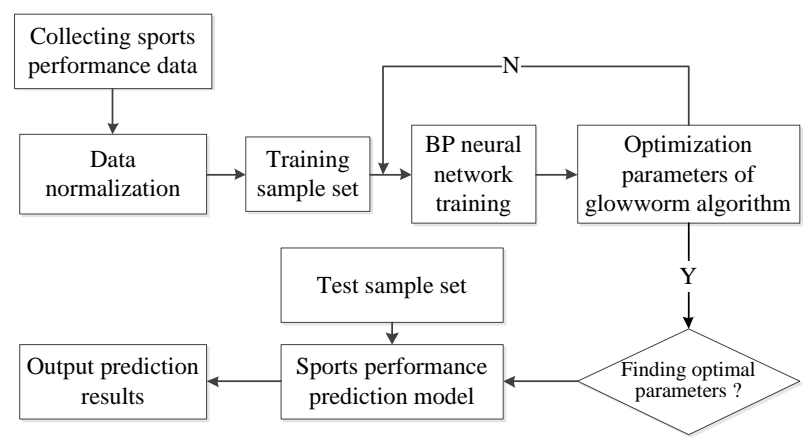

Fig. 1. Sports performance prediction model workflow.

\section{SiMULATION EXPERIMENT}

\section{A. Experimental Environment}

In order to test the validity of the model, 500 records of 100-meter race (unit:s) of Nanjing Forestry Police College were selected as the experimental objects. The model was realized by $\mathrm{VC}++6.0$ programming. The results of 100 -meter runs were shown in Fig. 2.

\section{B. Results and Analysis}

\section{1) Results of model predictions in this paper}

The training sample set was composed of 300 records of 100-meter results, and the BP neural network was used to train them. First, the connection weights and thresholds of the optimal BP neural network are found through the glowworm optimization algorithm. Secondly, the structure of BP neural network is determined according to the optimal connection weights and thresholds, and the performance prediction model of 100-meter race is established. Finally, the performance of the remaining 200 records of 100 -meter is predicted. The deviation between the predicted value and the actual value of the 100 meter score and the difference between them is shown in Figure 3. By analyzing the results of the 100-meter race in Fig. 3, it is found that the predicted results of the 100-meter race are very close to the measured ones, and the coincidence accuracy between them is quite high. This shows that a better performance prediction model for 100-meter race can be established by selecting the connection weights and thresholds of BP neural network with glowworm optimization algorithm. Moreover, the error between the predicted and measured results of 100-meter race is very small, which can be neglected completely, and the range of error variation is relatively narrow. The results verify the validity of the neural network sports performance prediction model optimized by glowworm algorithm. The prediction results are reliable and the prediction error is small.

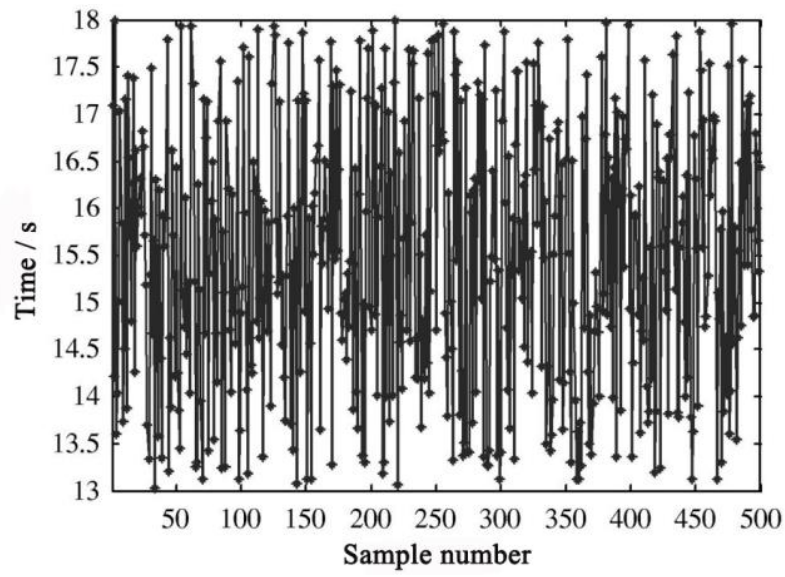

Fig. 2. Performance of 100 meters.

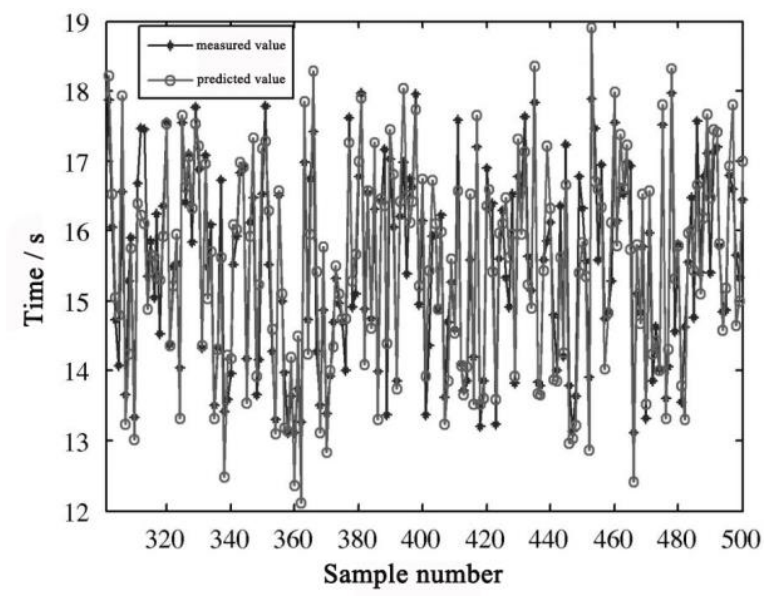

Fig. 3. Prediction of 100-meter race using glowworm optimized neural network.

\section{2) Comparison with other model predictions}

In order to analyze the superiority of the results of sports performance prediction of glowworm-optimized neural network, multiple linear regression, genetic algorithm and 
particle swarm optimization BP neural network were selected to carry out comparative experiments. The prediction accuracy was selected to evaluate the results of 100-meter race. The results are shown in Table I.

TABLE I: PREDICTION ACCURACY COMPARISON OF DIFFERENT MODELS

\begin{tabular}{lc}
\hline \hline \multicolumn{1}{c}{ Model } & Prediction accuracy \\
& $/ \%$ \\
\hline Multivariate linear regression model & 85.27 \\
BP neural network optimized by genetic algorithm & 90.24 \\
Particle swarm optimization BP neural network & 92.35 \\
glowworm optimization neural network & 95.78 \\
\hline \hline
\end{tabular}

The prediction accuracy of Table 1 can be concluded as follows:

1) Multivariate linear regression model has the lowest accuracy in predicting 100 -meter race performance, which shows that the model can not reflect the changing characteristics of 100-meter race performance.

2) Genetic algorithm optimizes BP neural network, particle swarm optimization BP neural network 100-meter race performance prediction accuracy is obviously better than the multiple linear regression model 100-meter race performance prediction accuracy. Because BP neural network is a kind of algorithm with strong nonlinear modeling ability, it can reflect the changing characteristics of $100 \mathrm{~m}$ race results and obtain better prediction results, but the prediction results of individual points are not ideal.

3) The predictive accuracy of the 100-meter running result of the glowworm-optimized neural network is higher than that of the BP neural network optimized by the genetic algorithm, and the BP neural network optimized by the particle swarm optimization. Because the glowworm-optimized algorithm can solve the problem that the genetic algorithm and the particle swarm optimization algorithm are difficult to find the connection weights and thresholds of the global optimal BP neural network, it is more accurate. It reflects the change trend of the results of the 100 meter race and gets a better result.

\section{CONCLUSION}

This paper studies the accurate prediction model of sports performance. In order to improve the prediction accuracy of sports performance, a prediction model of sports performance based on glowworm optimization neural network was proposed to solve the difficult problem of determining the connection weights and thresholds of BP neural network. Firstly, the training samples and test samples of the neural network are generated by pretreatment of the sports performance; secondly, the connection weights and thresholds of the BP neural network are determined by using the glowworm optimization algorithm, and the prediction model of the sports performance is established by learning the training samples; finally, the prediction effect is tested by specific simulation experiments. The results show that the glowworm optimized neural network improves the prediction accuracy of sports performance, and solves the limitations of other sports performance prediction models. The prediction results are more reliable, which can provide scientific basis for sports training decision-making.

\section{REFERENCES}

[1] Z. P. Wang and G. Sun, "Demonstration on the athletic achievements forecasting based on back propagation neural network," Journal of Nanjing Institute of Physical Education (Social Sciences), vol. 20, pp. 109-111, October 2006.

[2] B. J. Zhao, "Model system on main training targets of men's jumping," Journal of Xi'an Institute of Physical Education, vol. 18, pp. 81-82, January 2004.

[3] W. Zhou and J. Yan, "Analysis on gray correlatives of the hop step and jump technique of Chinese and foreign elite femate players," Journal of Jilin Institute of Physical Education, vol. 20, pp. 191-193, May 2015.

[4] L. Chang, G. M. Sun, and Y. C. Liu, "Research on sports achievements predictive based on the grey neural network," Value Engineering, vol. 20, pp. 64-65, May 2004.

[5] B. Long, "SVM-based prediction of Liu Xiang's achievements in 110 m hurdle," Journal of Tianjin University of Sport, vol. 24, pp. 330-333, October 2009.

[6] M. L Deng and Z. Zhang, "Research on the first three places in men's decathlon of Olympic using gray prediction model," Mathematics in Practice and Theory, vol. 39, pp. 44-49, August 2009.

[7] Z. Y. Li and S. Yan, "The nerve network model application in the forecast of sports result," Journal of Harbin Institute of Physical Education, vol. 27, pp. 116-119, April 2009.

[8] S. H. Yang, "Data mining based physical quality evaluation model for volleyball athletes," Modern Electronics Technique, vol. 40, pp. 119-122, June 2017.

[9] T. L. Cheng, S. Y. Zhou, and S. Li, "Extreme learning machine method for Short-term load forecasting," Electric Power Science and Engineering, vol. 29, pp. 24-29, July 2013.

[10] J. R. Chen, J. H. Chen, and Y. Wang, "Improved optimization algorithm on simulating fishing," Computer Engineering and Applications, vol. 47, pp. 47-50, March 2012.

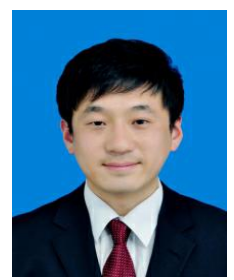

Fan Zhang was born in Jiangsu province, China. He received the $\mathrm{Ph}$.D. degree in sports science. In July 2016, he entered the post-doctoral research station of sports science in China Nanjing Normal University. He is currently an associate professor of Nanjing Forest Police College, China. His research interests include sports training and health promotion. He has published several articles and books. He has hosted or attended several Fund Projects of China. 